\title{
Vol. 67, No. 10
}

In the report "Notes from the Field: False-Negative Hepatitis B Surface Antigen Test Results in a Hemodialysis Patient - Nebraska, 2017," in the table on page 312, the testing instrument used by laboratory facility A should have read "ADVIA Centaur XP." 